\title{
A MULTICURVE TONE MAPPING OPERATOR FOR THE DISPLAY OF HIGH DYNAMIC RANGE IMAGE AND VIDEO
}

\author{
Min Chen and Guoping Qiu
}

School of Computer Science, University of Nottingham, UK

Keywords: high dynamic range imaging, tone mapping, display

\begin{abstract}
This paper presents a novel method for tone mapping high dynamic range images and videos for display in conventional low dynamic range devices. The new multi-curve method uses tone mapping curves that are adaptive to local luminance levels. The new method has the computational simplicity of curve based tone mapping methods and achieves results comparable to spatial operator methods. When applying the operator to render high dynamic range video, we introduce a bilateral style temporal filtering which successfully avoids undesirable shot noise and ghosting effects. It is also shown that our HDR tone mapping operator is a useful image enhancement method for standard format (8bits/pixel) images.
\end{abstract}

\section{Introduction}

The difference between the largest and the smallest luminance values in real world scenes, the dynamic range, can often exceed 4 orders of magnitude, much larger than that can be represented by standard image formats such as JPEG. As a result, today's cameras which in most cases use standard image formats have difficulty imaging high contrast scenes. One way to overcome this difficulty is to use high dynamic range imaging (HDRI), where the high dynamic range radiance maps use 32 bits/pixel or even higher bit depth formats. This means that the full dynamic range of the real world scenes can be faithfully recorded by the pixel values.

However, to be able to numerically represent the full dynamic range of the scenes is not enough. In most cases, we need to display the images. Most current visualization devices are low dynamic range (LDR) with a contrast ratio of a few orders magnitude. In order to reproduce HDR maps in LDR devices, mapping or tone reproduction techniques are used to map HDR values to LDR values. Tone reproduction (mapping) is a crucial step in the high dynamic range digital imaging workflow, because it doesn't matter how accurate the HDR maps may be, they have to be accurately and faithfully reproduced in the LDR devices. If the reproduction techniques fail, the whole workflow fails.

In the literatures, various tone mapping techniques have been developed for displaying high dynamic range images. They can be divided into two main categories, tone reproduction curve (TRC) based techniques and tone reproduction operator (TRO) based techniques [3]. Most TRC based methods use monotonic mapping functions derived from pixel distributions. In [4] the authors presented a tone mapping method based on modeling the relation between display brightness and real world luminance. Reference [5] is one of the most comprehensive TRC techniques and [6] presented an adaptive logarithmic curve method. More recently, a fast comprehensive tone mapping curve method with a closed form solution has been presented in [7, 8]. Tone Reproduction Operator (TRO) based techniques involve manipulating pixels locally to map the high dynamic range image for display. Breaking away from the monotonic mapping constraint enables the mapped image retains local contrasts and preserves more spatial details. Methods presented in [9-11] belong to this type of technique. Other tone mapping methods for HDR images include [12] where the authors attempted to incorporate traditional photographic technology in the digital domain for the reproduction of high dynamic range images and [13] where the authors presented a gradient domain high dynamic range image compression method.

In this paper, we present a novel method for tone mapping HDR images for display in LDR devices. From a theoretical rationale's point of view, our new method is inspired by the adaptation mechanism of the visual system and uses tone mapping curves that are adaptive to local luminance levels. To make full use of the limited dynamic range of the display devices, our method allows the same display level to represent different high dynamic range values if they are located in regions with different average luminance values (adaptation levels). From a practical engineering perspective, our method exploits the computational simplicity of TRC based method to achieve the better local detail preserving functionality of TRO based methods. To apply the operator to render video sequence, we introduce a temporal filtering scheme that is similar in spirit to bilateral filtering but applied to the temporal direction. We will show that the new filtering scheme can avoid shot noise and ghosting effects associated with simple temporal smoothing. Furthermore, it is also shown that our HDR tone mapping operator is also a useful image enhancement method and can achieve excellent results in enhancing standard format (8bits/pixel) images. 


\section{Adaptive Multi-curve HDR Image Mapping}

The visual world is hugely complex and the visual system is constantly exposed to scenes of huge dynamic ranges. Compared to the dynamic range of real world scenes we experience every day, the optimal response range of the visual cells is very limited. Apparently, the visual system performs various types of automatic control to cope with the high dynamic ranges and noisy environments to keep it within the optimal operating range. One such mechanism is called visual adaptation. It has been known that our visual system can adapt to environments with luminance ranging over more than 10 orders of magnitude.

Traditional curve-based global processing such as histogram equalization is known to have undesirable effects and researchers have developed adaptive local histogram processing method for image enhancement [14]. By adapting processing to local statistics, better results can be achieved. Our previous work has developed global histogram based approach to tone mapping high dynamic range images [7, 8], and we now present a natural extension to local processing.

\subsection{A Brightness Control Curve}

It has been demonstrated in $[7,8]$ that the overall brightness of a mapped HDR image can be controlled by a parameter $\tau$ using the curve of equation (1):

$$
D(I, \tau)=\left(D_{\max }-D_{\min }\right) \frac{\log (I+\tau)-\log \left(\mathrm{I}_{\min }+\tau\right)}{\log \left(\mathrm{I}_{\max }+\tau\right)-\log \left(\mathrm{I}_{\min }+\tau\right)}+D_{\text {min }}
$$

where $I_{\min }$ and $I_{\max }$ are the minimum and maximum luminance of the scene, and $D_{\max }$ and $D_{\min }$ are the minimum and maximum luminance of the visualization devices. The smaller $\tau$ is, the brighter the image looks, and vice versa. Furthermore, with $\tau$ increasing, local contrast in brighter area is higher. Fig. 1 shows an example of the curves of (1) for different $\tau$ 's and the mapped LDR images using these curves.

From Fig.1, we can see that, a bigger $\tau$ will enable high luminance areas such as the window on the roof to be displayed clearly; on the other hand, in order to make dark areas, such as the upper right region in the image, to be displayed clearly, we need a smaller $\tau$. Motivated by such observations, we reason that for a given image region, it is possible to find an appropriate value of $\tau$ to generate a mapping curve according to (1) to map the pixels within that region such that details and contrast of the region can be clearly displayed.

\subsection{A Simple Multi-curve Tone Mapping Operator}

Based on the observation in Fig. 1, a simple approach is to divide the image into blocks and each mapped by its own mapping curve computed according to (1). For a given block $\boldsymbol{B}$ the mapping curve's $\tau=\tau(\mathrm{B})$ should be a function of the average intensity of the block. We found that setting $\tau(B)$ to the average of logarithm intensity of the block works well for all the images we tested.
Not surprisingly, this simple mapping method will cause visual artifacts (see the left image of Fig. 3) because a lower luminance pixel can be displayed brighter than a higher luminance pixel or vice versa; or the same luminance value could be mapped to different display levels as illustrated in Fig. 2. With reference to Fig. 2, two adjacent pixels $a$ and $b$ in two neighboring blocks $\mathrm{A}$ and $\mathrm{B}$. Because the average intensities of $A$ and $B$ are different, the mapping curves used to map $a$ and $b$ will be different. Artifacts will occur if $a=b$ as shown in the buttom of Fig.2, because the same value has been mapped to different values by using different tone mapping curves, in this case $\mathrm{D}(\mathrm{a})>\mathrm{D}(\mathrm{b})$. To remove such undesirable effect, we use bilateral filter to calculate the local brightness level for each pixel. As shown in the top right, a and $b$ have the similar average brightness, hence $a$ and $b$ are mapped by the same tone mapping curve and have the same display value. Fig. 3 shows the effect of applying such a process to the result of the new multi-curve tone mapping operator.
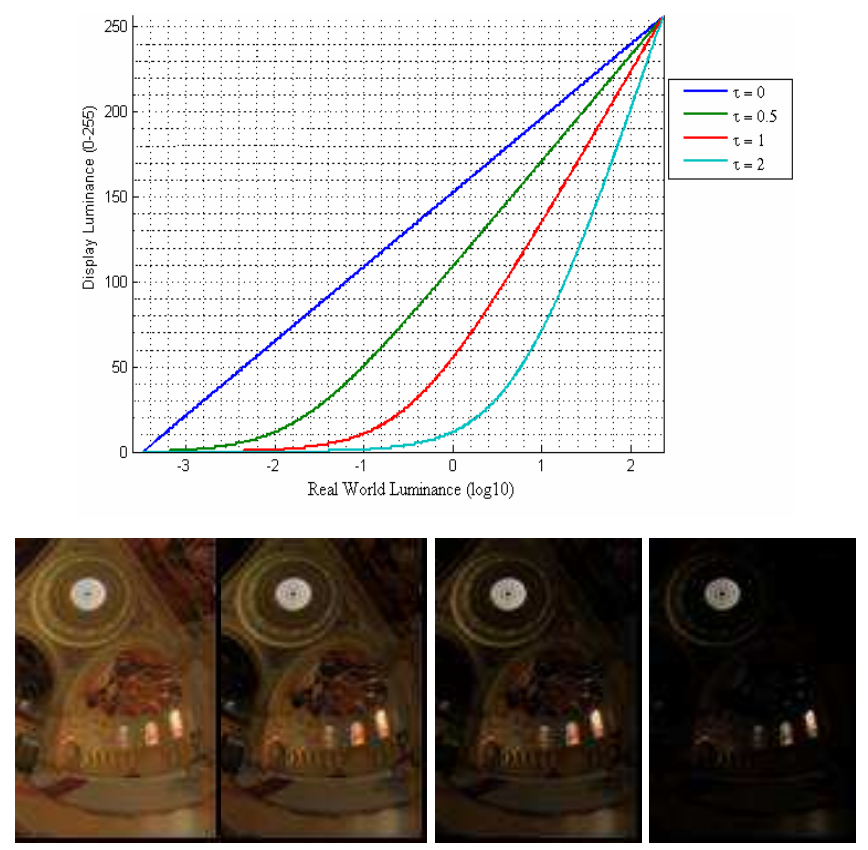

Fig.1 Brightness control curves (top) and mapped LDR images using these curves (bottom, from left to right, $\tau=0$, $\tau=0.5, \tau=1, \tau=2)$. The HDR image is courtesy of Paul Debevec 


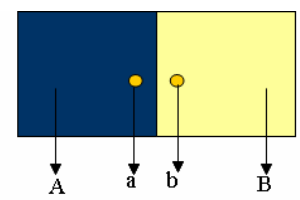

$\tau(\mathrm{a})=\tau(\mathrm{A})>\tau(\mathrm{B})=\tau(\mathrm{b}), \mathrm{a}=\mathrm{b}$

After tone mapping

$\mathrm{D}(\mathrm{a})>\mathrm{D}(\mathrm{b})$

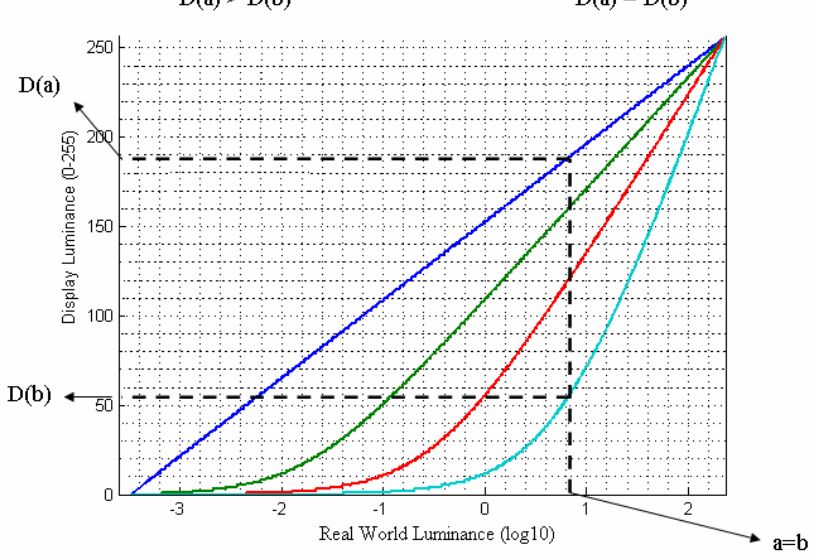

Fig.2. The cause of artifacts and ways to remove them. In practice, the operation is performed as a filtering process.
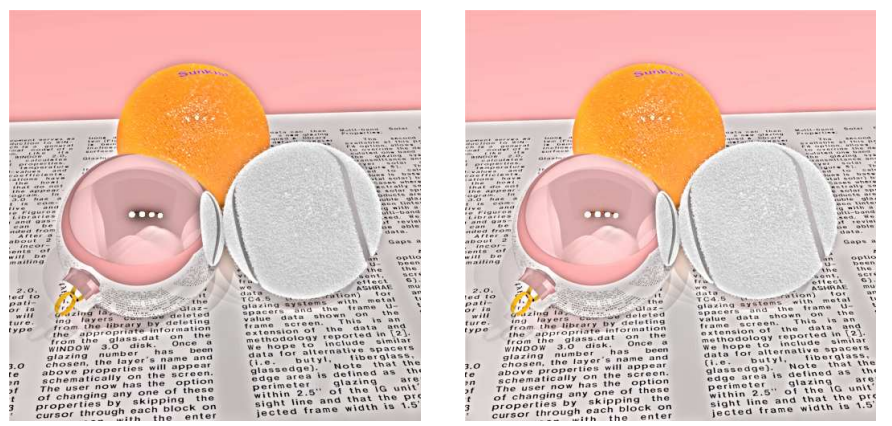

Fig.3. Left: output by the multi-curve mapping operator. It is seen that all features are clearly displayed. In fact, the display has very high contrasts; however, the contrasts are false thus resulting in artifacts such as the dark shadows surrounding the specular highlights. Right: output of applying a filtering process. It is seen that the visual artifacts has been removed while details are well preserved.

\subsection{An Improved Brightness Control Curve}

As shown in Fig.1, with a larger $\tau$, high luminance values are mapped to a wider display range resulting higher contrasts. For a smaller $\tau$, there is not enough contrast at the low end of the input range. Ideally, we want the display to have similar contrasts regardless of its brightness, which means we would like to have curves with similar ratios in its operating range. We modify the curve in (1) by making the base of the logarithm changing with the adaptation level and the pixel intensity:

$D(I, \tau)=\left(D_{\max }-D_{\min }\right) \frac{\log _{b(\tau)}(I+\tau)-\log _{b(\tau)}\left(\operatorname{Im}_{\mathrm{m} i n}+\tau\right)}{\log _{b(\tau)}(\operatorname{Im} a x+\tau)-\log _{b(\tau)}(\operatorname{Im} i n+\tau)}+D_{\text {min }}$

The base of the logarithm is chosen as $b(\tau)=2+\frac{8 \tau}{\tau_{\max }}+\frac{8 I\left(\tau_{\max }-\tau\right)}{I_{\max } \tau_{\max }}$

where $\tau_{\max }=\log \left(I_{\max }\right)$. We set the base range from 2 (when $\tau$ $=0$ ) to 10 (when $\tau=\tau_{\max }$ ). For the same $\tau$, a larger $I$ will result in a larger base, for the same $I$, a larger $\tau$ will also result in a larger base. The curve of equation (2) for different $\tau$ is shown in Fig. 4. It is seen that for a smaller $\tau$, the output contrast is enhanced compared to (1). Furthermore, the ratios in the linear intervals of the curves are similar meaning that the contrast of the mapped image at different adaptation level will be similar. Such curve will ensure that local regions have similar contrast regardless of their brightness. We found that this is an important factor that makes our technique works.

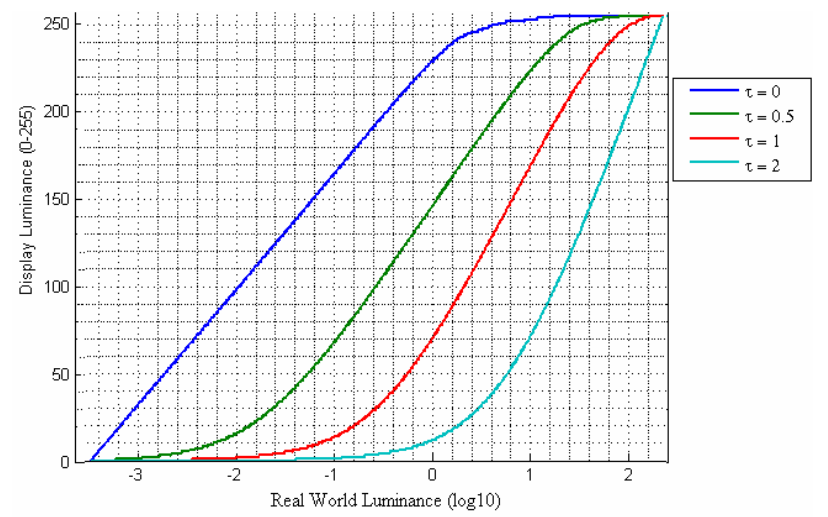

Fig.4 Improved brightness control curves (2) for different $\tau$ 's.

\section{Still HDR Image Mapping Results}

We have tested our algorithm on many high dynamic range images. In all our results, images are divided into $32 \times 32$ blocks (other block sizes work similarly well) and mapped using the curve of (2). Fig. 5 shows two examples of our results and results of a representative method from the literature. More results and comparison with many other methods can be viewed at this URL

\section{http://www.viplab.cs.nott.ac.uk/hdri}

One of the common practices in evaluating tone mapping operators is through subjective visual judgment. Based on such evaluation, we found that our method is very competitive to state of the art.

Our method can also be applied to the enhancement of ordinary ( 8 bits/pixel) images. Fig. 6 shows examples of image enhancement using our new method and comparison with the gradient domain method [13].

\section{Rendering HDR Video for Display}

Our multicurve tone mapping operator can be easily applied to render high dynamic range video for display (in lowdynamic range devices). A straightforward application is to map individual frame using the operator described in Section 2. However, it is very likely to produce shot noise. Dubois and Sabri [17] proposed a nonlinear temporal filter using 
motion compensation for shot noise reduction. Pixels are temporally combined using a recursive low-pass temporal filter by the incorporation of motion estimation and compensation. Jostschulte et al [18] presented a spatialtemporal noise reduction system, which filters video both spatially and temporally while preserving edge. Bennett and McMillan [19] developed a robust LDR video enhancement system using per-pixel virtual exposure. Since LDR images/videos enhancement has much in common with HDR acquisition, this method is also described for HDR compression. Kang et al [16] who developed a HDR video capturing system, proposed a simple HDR video tone mapping method. HDR frames are spatially tone mapped by Reinhard's tone mapping operator [12], and log-averaged in temporal direction to prevent temporal artefacts such as flicking.

We first render each individual frame independently using the new multicurve operator, we then apply the following 3-D filtering operation to the video

$$
D^{\prime}\left(I_{x, y, t}\right)=\frac{\sum_{m=-N}^{N} D\left(I_{x, y, t+m}\right) S_{t}(m)}{\sum_{m=-N}^{N} S_{t}(m)}
$$

where $(\mathrm{x}, \mathrm{y}, \mathrm{t})$ is the pixel's spatial and temporal co-ordinates, $D\left(I_{\mathrm{x}, \mathrm{y}, \mathrm{t}}\right)$ the display value mapped frame by frame using the operator described earlier and $D^{\prime}\left(I_{\mathrm{x}, \mathrm{y}, \mathrm{t}}\right)$ is the final display value after temporal smoothing. The similarity function $S_{t}$ is defined as

$$
S_{t}(m)=\exp \left(\frac{m^{2}}{\sigma_{d}^{2}}\right) \exp \left(\frac{\|H(t+m)-H(t)\|^{2}}{\sigma_{f}^{2}}\right)
$$

where $\mathrm{H}(\mathrm{t})$ is the histogram of frame $t$.

\section{Video Display Results}

We tested our HDR video tone mapping approach for several HDR video clips created by Microsoft Research. In Fig. 7 it is shown that compared with simple Gaussian smoothing in the temporal direction, "ghosting" artifact is successfully avoided by applying the temporal similarity function the filtering. In Fig. 8, we show that shot noise can be avoided by using a bilateral style filtering in the temporal direction. The rendered video can also be seen in this website

http://www.viplab.cs.nott.ac.uk/hdri

\section{Concluding Remarks}

In this paper, we have introduced a multicurve tone mapping operator for display high dynamic range and video. For rendering video, we also introduced a temporal filtering scheme that successfully avoided shot noise and ghosting effects. Compared our new method with many existing ones, our has the advantages that it has the computational simplicity of curve based methods and achieve results that is comparable with local operators but with lower complexity and easier to implement. We have also shown that the bilateral style temporal filtering scheme can avoid artifacts such as shot noise and ghosting effects of traditional linear temporal smoothing.
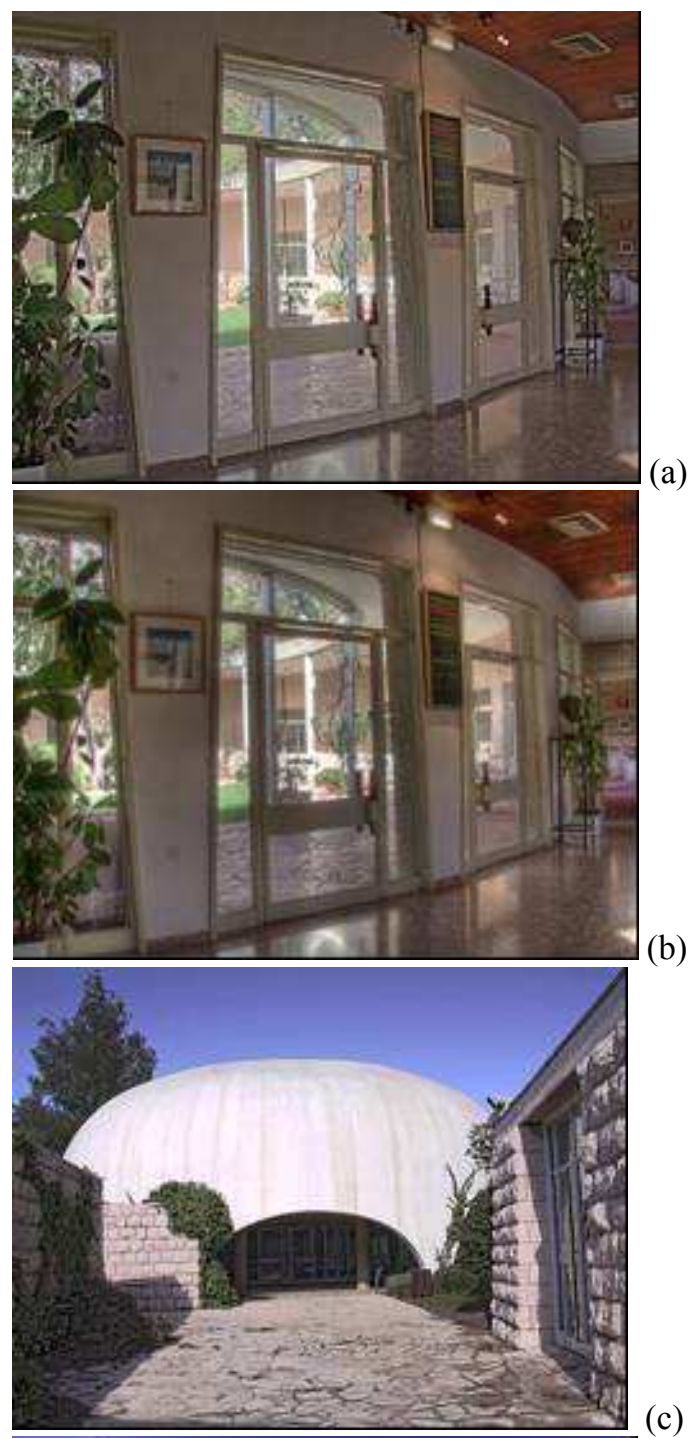

(c)

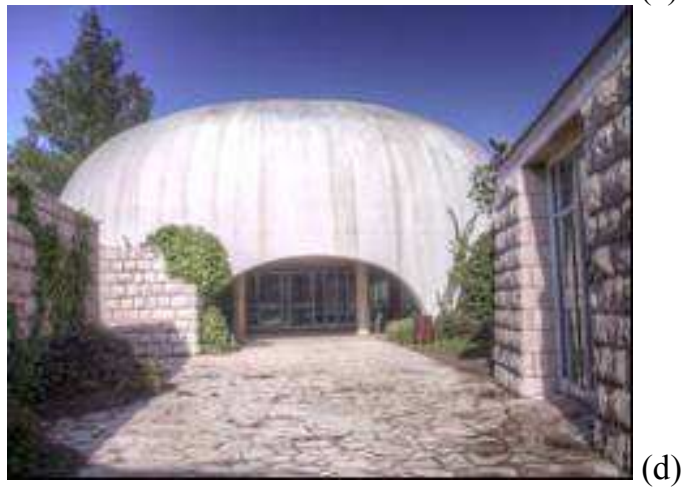

Fig.5 (a) and (c) our results, (b) and (d) result of [13]. Note the severe halo artifacts between the tree and the sky in (d). Image data courtesy of R. Fattal, D. Lischinski and M. Werman [13]. 

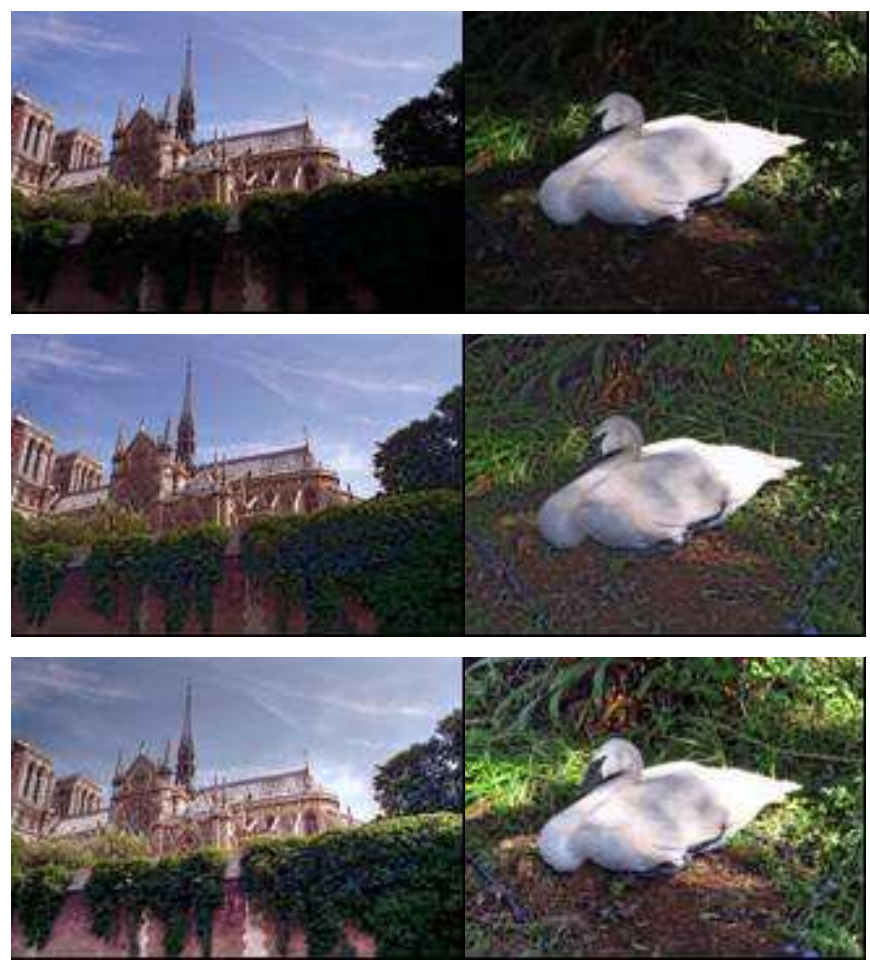

Fig. 6 Image enhancement results. Tow row: original 8 bit/pixel images. Middle row: enhancement results of our method. Bottom row: results of [13].

Simple temporal smoothing method (e.g. average or Gaussian smoothing) can reduce shot noise; however, it will introduce "ghosting" artefacts as well. By applying a bilateral style filter, "ghosting" artefacts are successfully reduced.

\section{References}

[1] P. E. Debevec and J. Malik, "Recovering high dynamic range radiance maps from photographs", In Proceedings of SIGGRAPH'97, pp. $369-378$

[2] T. Mitsunaga and S. K. Nayar, "High dynamic range imaging: Spatially varying pixel exposures", Proc. CVPR'2000, vol. 1, pp. 472-479, 2000

[3] J. DiCarlo and B. Wandell, "Rendering high dynamic range images", Proc. SPIE, vol.3965, pp. 392 - 401, 2001

[4] J. Tumblin and H. Rushmeier, "Tone reproduction for realistic images", IEEE Computer Graphics and Applications, vol. 13, pp. 42 - 48, 1993

[5] G. Ward Larson, H. Rushmeier and C. Piatko. A visibility matching tone reproduction operator for high dynamic range scenes. IEEE Trans on Visualization and Computer Graphics, vol. 3, pp. 291 - 306, 1997
[6] F. Drago, K. Myszkowski, T. Annen and N. Chiba, "Adaptive Logarithmic Mapping For Displaying High Contrast Scenes", The Journal of Computer Graphics Forum, Vol.22, No, 3, pp. 419-426, 2003

[7] J Duan, G. Qiu and M. Chen, "Comprehensive Fast Tone Mapping for High Dynamic Range Image Visualization", Pacific Graphics 2005

[8] G Qiu, J. Guan, J. Duan and M. Chen, "Tone mapping for HDR image using optimization - A new closed form solution", ICRP 2006

[9] K. Chiu, M. Herf, P. Shirley, S. Swamy, C. Wang and K. Zimmerman, "Spatially nonuniform scaling functions for high contrast images", Proc. graphics Interface'93, pp. $245-253,1993$

[10] J. Tumblin and G. Turk, "LCIS: A boundary hierarchy for detail preserving contrast reduction", In Proc. of ACM SIGGRAPH'99, pp. 83-90.

[11] F. Durand and J. Dorsey, Fast bilateral filtering for the display of high-dynamic-range images. ACM Trans. Graph. (special issue SIGGRAPH 2002) 21, 3, 257-266, 2002

[12] E. Reinhard, M. Stark. P. Shirley and J. Ferwerda, "Photographic tone reproduction for digital images", In Proceedings of SIGGRAPH 2002, pages 267-276, 2002.

[13] R. Fattal, D. Lischinski and M. Werman, "Gradient domain high dynamic range compression", In Proceedings of SIGGRAPH 2002, pages 249-256, 2002.

[14] J. Y. Kim, L. S. Kim, S. H. Hwang, "An advanced contrast enhancement using partially overlapped subblock histogram equalization", Circuits and Systems for Video Technology, IEEE, Vol.11, 2001 pp: 475 -484

[15] C. Tomasi, R. Manduchi "Bilateral filtering for gray and color images" In Proc. of International Conference on Computer Vision, IEEE (1998) 839-846

[16] S. B. Kang, M. Uyttendaele, S. Winder, and R. Szeliski, 2003. High Dynamic Range Video, ACM Transactions on Graphics, 22, 3, 319-325

[17] E. Dubiois, and S. Sabri, "Noise Reduction in Image Sequences Using Motion-Compensated Temporal Filtering", IEEE Transactions on Communications, 32, 7, 826-831, 1984

[18] K. Jostschulte, A. Amer, M. Schu, and H. Scroder, "Perception Adaptive Temporal TV-Noise Reduction Using Contour Preserving Prefilter Techniques", IEEE Transactions on Consumer Electronics, 44, 3 (August), 1091-1096, 1998.

[19] E. P. Bennett and L. McMillan "Video Enhancement Using Per-Pixel Virtual Exposures", SIGGRAPH 2005 


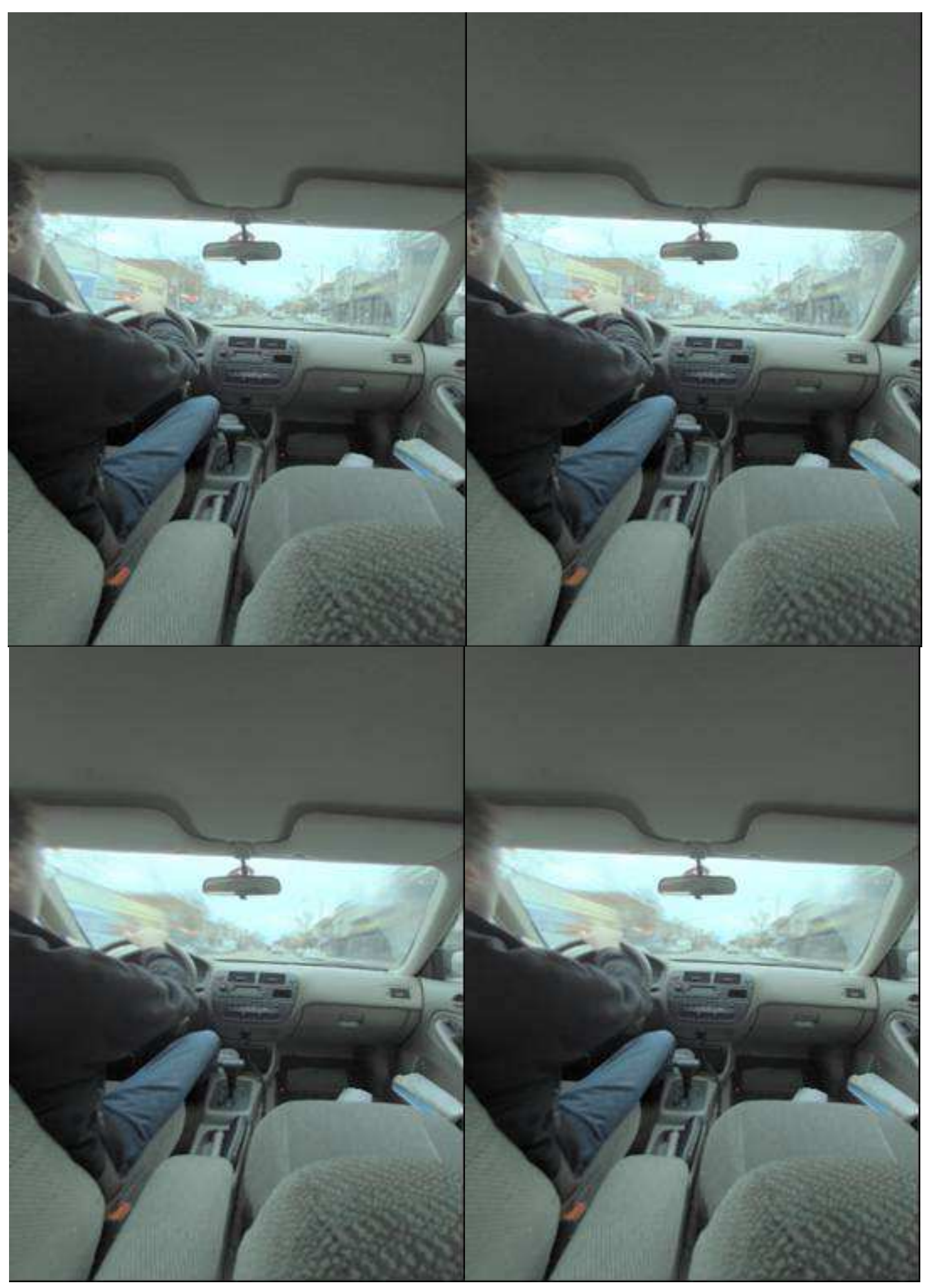

Fig. 7. Capitol Hill Drive HDR video sequence, courtesy of S. B. Kang. Top row: frames 14 and 16 rendered by the multicurve operator with a bilateral style post processing (4) and (5) applied to the temporal direction. Bottom row: frames 14 and 16 mapped by the multicurve operators but with simple Gaussian smoothing applied in the temporal direction. It is seen that the areas outside the car windscreen appear blur when simple Gaussian was used while they are much clearer when (4) and (5) were used. 


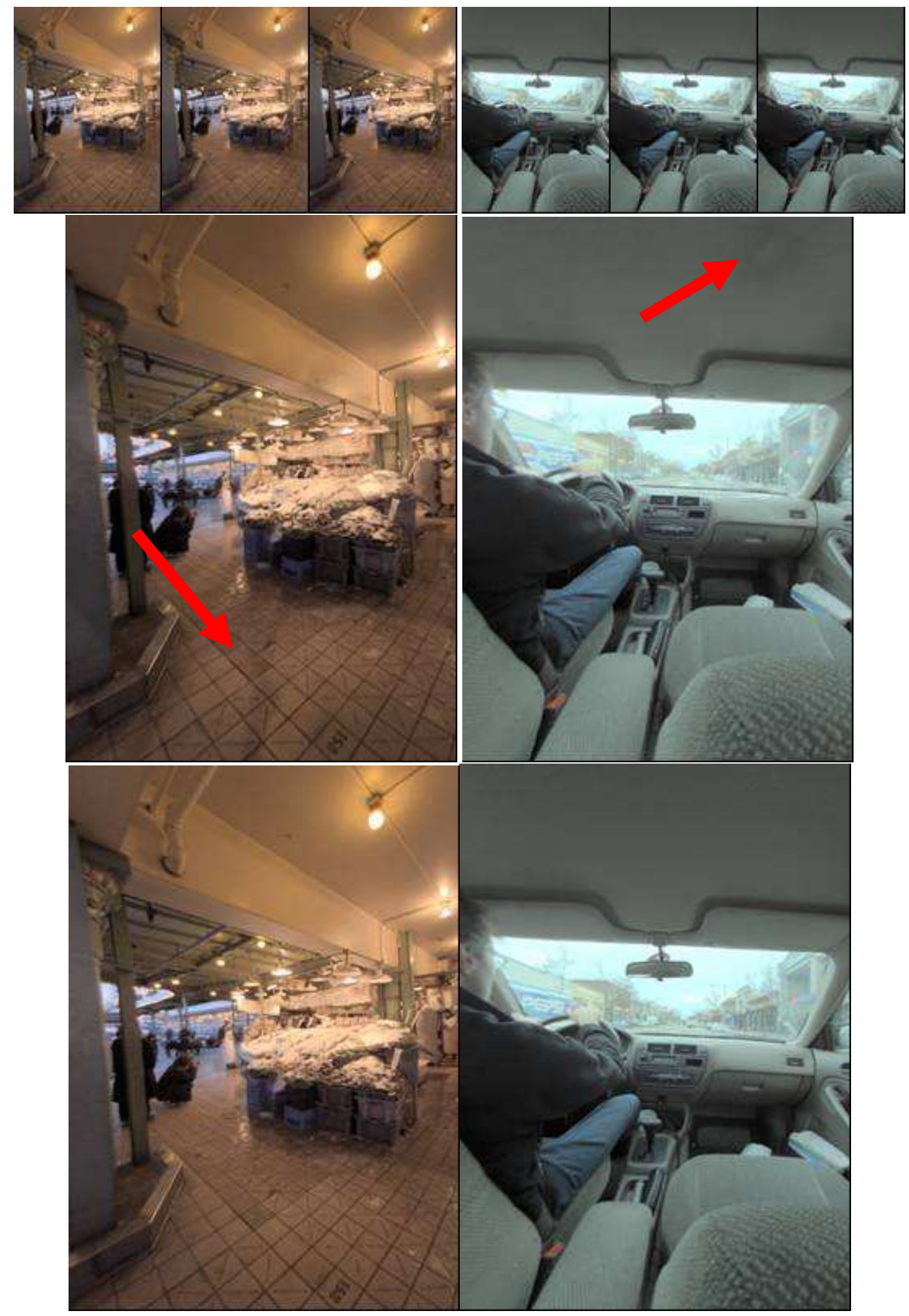

Fig.8 Top row: examples of individually rendered frames. Middle row: temporal processing using simple Gaussian smoothing, it is seen that artefacts have appeared (pointed by the read arrows). Bottom row: temporal processing with our bilateral style filter of (4) and (5), it is seen such artefacts have disappeared. 